\title{
Whitney Multiapproximation
}

\section{Eman Samir Bhaya}

\author{
Mayada Ali Kareem
}

University of Babylon, College of Education for science pure, Mathematics Department, Babil. Iraq

Keywords: Whitney theorem, Multiapproximation, Lagrange polynomial.

\begin{abstract}
In this article we prove that Whitney theorem for the value of the best multiapproximation of a function $f \in L_{p}\left([a, b]^{d}\right), 0<p<\infty$ by algebraic multipolynomial $p_{m-1}$ of degree $\leq m-1$.
\end{abstract}

\section{Introduction, definitions and main result}

Whitney theorem has applications in many areas and has been further generalized to various classes of function and other approximating spaces .

Whitney theorem was proved by Burkill [1] when $(k=2, p=\infty)$ and Storozhenko [2] when $(0<p<1)$.

In [3], [4] Whitney proved that if $f \in C([a, b])$ then $E_{k-1}(f)_{[a, b]} \leq W_{k} \omega_{k}\left(f, \frac{b-a}{k},[a, b]\right)$ where $W_{k}=$ const depends only on $k$.

In 2003 E.S. Bhaya [5] proved the following theorem by using Whitney theorem of interpolatory type for $k$-monotone functions for K. A. Kopotun.

Theorem A: Let $m, k \in N, m<k$ and $f \in \Delta^{k} \cap W_{p}^{m}(I)$. Then for any, $n \geq k-1$, there exists apolynomial $p_{n} \in \Pi_{n}$ such that for any $p<1$

$$
\left\|f^{(j)}-p_{n}^{(j)}\right\|_{p} \leq c(p, k) \omega_{k-j}^{\varphi}\left(f^{(j)}, n^{-1}, I\right)_{p} \text { for } j=1, \ldots, m
$$

In 2004 S.Dekel and D.Leviatan [6] proved the following Whitney estimate. 
Theorem B: Given $0<p \leq \infty, r \in N$, and $\quad d \geq 1$, there exists a constant $C(d, r, p)$, depending only on the three parameters, such that for every bounded convex domain $\Omega \subset R^{d}$, and each function $f \in L_{p}(\Omega)$,

$$
E_{r-1}(f, \Omega)_{p} \leq C(d, r, p) \omega_{r}(f, \operatorname{diam}(\Omega), \Omega)_{p}
$$

where $E_{r-1}(f, \Omega)_{p}$ is the degree of approximation by polynomials of total degree $r-1$, and $\omega_{r}(f, \cdot)_{p}$ is the modulus of smoothness of order $r$.

In 2011 Dinh Dung and Tino Ullrich [7] proved the following Whitney type inequalities

Theorem C: Let $1 \leq p \leq \infty, r \in \mathrm{N}^{d}$. then there is a constant $\mathrm{C}$ depending only on $r, d$ such that for every $f \in L_{p}(Q)$

$$
\left(\sum_{e \subset[d]} \prod_{i \in e} 2^{r_{i}}\right)^{-1} \Omega(f, \delta, Q)_{p, Q} \leq E_{r}(f)_{p, Q} \leq C \Omega(f, \delta, Q)_{p, Q}
$$
of $Q$.

Where $Q:=\left[a_{1}, b_{1}\right] \times \ldots \times\left[a_{d}, b_{d}\right]$ and $\delta=\delta(Q):=\left(b_{1}-a_{1}, \ldots, b_{d}-a_{d}\right)$ is the size

For the proof our main result we need the following definitions :

Let us introduce a new version of Lagrange polynomial on $R^{d}$, and call it a Lagrange multipolynomial .

\section{Definition 1.1.}

A Lagrange multipolynomial $L(x, f)=L\left(\left(x_{1}, x_{2}, \ldots, x_{d}\right) ; f\right)$

$L\left(\left(x_{1}, x_{2}, \ldots, x_{d}\right) ; f\right)=L\left(\left(x_{1}, \ldots, x_{d}\right) ; f ;\left(x_{01}, \ldots, x_{0 d}\right),\left(x_{11}, \ldots, x_{1 d}\right), \ldots,\left(x_{m 1}, \ldots, x_{m d}\right)\right)$

that interpolates a function $f$ at points $x_{0}=\left(x_{01}, \ldots, x_{0 d}\right), x_{1=}\left(x_{11}, \ldots, x_{1 d}\right), \ldots, x_{m}=$ $\left(x_{m 1}, \ldots, x_{m d}\right)$ (interpolation nodes) is defined as an algebraic multipolynomial of at most $m t h$ order that takes the same values at these points as the function $f$, that is

$$
L\left(x_{i} ; f\right)=L\left(\left(x_{i 1}, \ldots, x_{i d}\right) ; f\right)=f\left(\left(x_{i 1}, \ldots, x_{i d}\right)\right)
$$

where $i=0, \ldots, m$.

Example, for $m=1$ we have 


$$
\begin{aligned}
& L\left(x ; f ; x_{0}, x_{1}\right)=L\left(\left(x_{1}, \ldots, x_{d}\right) ; f ;\left(x_{01}, \ldots, x_{0 d}\right),\left(x_{11}, \ldots, x_{1 d}\right)\right) \\
& =\frac{\left(x_{1}-x_{11}\right) \ldots\left(x_{d}-x_{1 d}\right)}{\left(x_{01}-x_{11}\right) \ldots\left(x_{0 d}-x_{1 d}\right)} f\left(\left(x_{01}, \ldots, x_{0 d}\right)\right)+\frac{\left(x_{1}-x_{01}\right) \ldots\left(x_{d}-x_{0 d}\right)}{\left(x_{11}-x_{01}\right) \ldots\left(x_{1 d}-x_{0 d}\right)} f\left(\left(x_{11}, \ldots, x_{1 d}\right)\right) \\
& =f\left(x_{01}, \ldots, x_{0 d}\right)+\frac{f\left(\left(x_{11}, \ldots, x_{1 d}\right)\right)-f\left(\left(x_{01}, \ldots, x_{0 d}\right)\right)}{\left(x_{11}-x_{01}\right) \ldots\left(x_{1 d}-x_{0 d}\right)}\left(\left(x_{1}-x_{01}\right) \ldots\left(x_{d}-x_{0 d}\right)\right) \\
& \quad \text { where } x_{0 j} \neq x_{1 j} \quad, \quad j=1, \ldots, d
\end{aligned}
$$

\section{Definition 1.2.}

$$
\begin{aligned}
& \text { Let } I_{k}(x)=I_{k}\left(\left(x_{1}, \ldots, x_{d}\right)\right)=I_{k}\left(\left(x_{1}, \ldots, x_{d}\right) ;\left(x_{01}, \ldots x_{0 d}\right), \ldots,\left(x_{m 1}, \ldots, x_{m d}\right)\right) \\
& =\prod_{\substack{i=0 \\
k \neq i}}^{m} \frac{\left(x_{1}-x_{i 1}\right) \ldots\left(x_{d}-x_{i d}\right)}{\left(x_{k 1}-x_{i 1}\right) \ldots\left(x_{k d}-x_{i d}\right)}, \quad k=0, \ldots, m
\end{aligned}
$$

a new version of fundamental Lagrange multi polynomials.

We set

$$
\begin{aligned}
p(x) & =p\left(\left(x_{1}, \ldots, x_{d}\right)\right), x \in R^{d} \\
& =\left(\left(x_{1}-x_{01}\right) \ldots\left(x_{d}-x_{0 d}\right)\right)\left(\left(x_{1}-x_{11}\right) \ldots\left(x_{d}-x_{1 d}\right)\right) \ldots\left(\left(x_{1}-x_{m 1}\right) \ldots\left(x_{d}-x_{m d}\right)\right) .
\end{aligned}
$$

And note that

$$
\begin{aligned}
\dot{p}\left(\left(x_{k 1}, \ldots, x_{k d}\right)\right) & =\lim _{\substack{x_{j} \rightarrow x_{k j} \\
j=1, \ldots, d}} \frac{p\left(\left(x_{1}, \ldots, x_{d}\right)\right)}{\left(\left(x_{1}-x_{k 1}\right) \ldots\left(x_{d}-x_{k d}\right)\right)} \\
& =\lim _{\substack{x_{j} \rightarrow x_{k j} \\
j=1, \ldots, d}} \prod_{i=0}^{m}\left(\left(x_{1}-x_{i 1}\right) \ldots\left(x_{d}-x_{i d}\right)\right) \\
& =\prod_{i=0}^{m}\left(\left(x_{k 1}-x_{i 1}\right) \ldots\left(x_{k d}-x_{i d}\right)\right) .
\end{aligned}
$$

Therefore , for any $k=0, \ldots, m$, the new version of the fundamental Lagrange multipolynomials are represented in the form 


$$
\begin{aligned}
& I_{k}\left(\left(x_{1}, \ldots, x_{d}\right)\right)=I_{k}\left(\left(x_{1}, \ldots, x_{d}\right) ;\left(x_{01}, \ldots, x_{0 d}\right),\left(x_{11}, \ldots, x_{1 d}\right), \ldots,\left(x_{m 1}, \ldots, x_{m d}\right)\right) \\
& =\frac{p\left(\left(x_{1}, \ldots, x_{d}\right)\right)}{\left(\left(x_{1}-x_{k 1}\right) \ldots\left(x_{d}-x_{k d}\right)\right) \dot{p}\left(\left(x_{k 1}, \ldots, x_{k d}\right)\right)}, \\
& \quad \text { where } x_{j} \neq x_{k j} \quad, \quad j=1, \ldots, d \quad, \quad k=0, \ldots, m .
\end{aligned}
$$

Let $\delta_{i, k}$ denote the Kronecker symbol, which is equal to 1 for $i=k$ and to 0 otherwise .

It follows from the obvious equality $I_{k}\left(x_{i 1}, \ldots, x_{i d}\right)=\delta_{i, k}, i, k=0, \ldots, m$, that the Lagrange multipolynomial exists and is represented by the relation

$$
\begin{aligned}
& L\left(\left(x_{1}, \ldots, x_{d}\right) ; f ;\left(x_{01}, \ldots x_{0 d}\right), \ldots,\left(x_{m 1}, \ldots, x_{m d}\right)\right) \\
& =\sum_{k=0}^{m} f\left(\left(x_{k 1}, \ldots, x_{k d}\right)\right) I_{k}\left(\left(x_{1}, \ldots, x_{d}\right) ;\left(x_{01}, \ldots x_{0 d}\right), \ldots,\left(x_{m 1}, \ldots, x_{m d}\right)\right)
\end{aligned}
$$

\section{Definition 1.3.}

The expression $\left[\left(x_{01}, \ldots, x_{0 d}\right),\left(x_{11}, \ldots, x_{1 d}\right), \ldots,\left(x_{m 1}, \ldots, x_{m d}\right) ; f\right]$

is called the divided difference of order $m$ for the function $f$ at the points $x_{0}=\left(x_{01}, \ldots, x_{0 d}\right), x_{1}=\left(x_{11}, \ldots, x_{1 d}\right), \ldots, x_{m}=\left(x_{m 1}, \ldots, x_{m d}\right)$

For example

$$
\begin{aligned}
{\left[x_{0}, x_{1} ; f\right] } & =\frac{f\left(\left(x_{01}, \ldots, x_{0 d}\right)\right)}{\left(x_{01}-x_{11}\right) \ldots\left(x_{o d}-x_{1 d}\right)}+\frac{f\left(\left(x_{11}, \ldots, x_{1 d}\right)\right)}{\left(x_{11}-x_{01}\right) \ldots\left(x_{1 d}-x_{0 d}\right)} \\
& =\frac{f\left(\left(x_{01}, \ldots, x_{0 d}\right)\right)-f\left(\left(x_{11}, \ldots, x_{1 d}\right)\right)}{\left(x_{01}-x_{11}\right) \ldots\left(x_{o d}-x_{1 d}\right)}
\end{aligned}
$$

Let $\left[x_{0} ; f\right]=\left[\left(x_{01}, \ldots, x_{0 d}\right) ; f\right]=$ $f\left(\left(x_{01}, \ldots, x_{0 d}\right)\right)$. 


\section{Definition 1.4.}

The expression

$\Delta_{h}^{m}\left(f ;\left(x_{01}, \ldots, x_{0 d}\right)\right):=\sum_{k=0}^{m}\left((-1)^{m-k}\left(\begin{array}{c}m \\ k\end{array}\right)\right)^{d} f\left(\left(x_{01}+k h_{1}, \ldots, x_{0 d}+k h_{d}\right)\right)$

where $d \in N$ chosen so that $(-1)^{m-k}=(-1)^{d}$

is called the multi mth difference of the function $f \in L_{p}\left([a, b]^{d}\right), 0<p<\infty$ at the point $x_{0}=\left(x_{01}, \ldots, x_{0 d}\right)$ with step $h=\left(h_{1}, \ldots, h_{d}\right)$.

Denote $\Delta_{h}^{0}\left(f ;\left(x_{01}, \ldots, x_{0 d}\right)\right)=f\left(\left(x_{01}, \ldots, x_{0 d}\right)\right)$ and $\Delta_{0}^{m}\left(f ;\left(x_{01}, \ldots, x_{0 d}\right)\right)=0$.

\section{Our main result is:}

\section{Theorem 1.1.}

If $f \in L_{p}\left([a, b]^{d}\right), 0<p<\infty$, then

$$
E_{m-1}(f)_{L_{p}[a, b]^{d}} \leq C(p, m, d) \omega_{m}\left(f ; h ;[a, b]^{d}\right)_{p}
$$

where $h=\left(h_{1}, \ldots, h_{d}\right)$.

Now to prove our theorem we need the lemmas and theorems which will be stated and proved in the following sections :

\section{Divided differences}

Let us define the difference

$f\left(\left(x_{1}, \ldots, x_{d}\right)\right)-L\left(\left(x_{1}, \ldots, x_{d}\right) ; f ;\left(x_{01}, \ldots, x_{0 d}\right), \ldots,\left(x_{m 1-1}, \ldots, x_{m d-1}\right)\right)$,

by the product $\left(\left(x_{1}-x_{01}\right) \ldots\left(x_{d}-x_{0 d}\right)\right) \ldots\left(\left(x_{1}-x_{m 1-1}\right) \ldots\left(x_{d}-x_{m d-1}\right)\right)$

Using (4) and (5), we represent the quotient at the points $x_{1}=x_{m 1}, \ldots, x_{d}=x_{m d}$ as follows : 


$$
\begin{aligned}
& \frac{f\left(\left(x_{m 1}, \ldots, x_{m d}\right)\right)-L\left(\left(x_{m 1}, \ldots, x_{m d}\right) ; f ;\left(x_{01}, \ldots, x_{0 d}\right), \ldots,\left(x_{m 1-1}, \ldots, x_{m d-1}\right)\right)}{\prod_{k=0}^{m-1}\left(\left(x_{m 1}-x_{k 1}\right) \ldots\left(x_{m d}-x_{k d}\right)\right)} \\
& =\sum_{k=0}^{m} \frac{f\left(\left(x_{k 1}, \ldots, x_{k d}\right)\right)}{\prod_{\substack{i=0 \\
i \neq k}}^{m}\left(\left(x_{k 1}-x_{i 1}\right) \ldots\left(x_{k d}-x_{i d}\right)\right)} \\
& =\left[\left(x_{01}, \ldots, x_{0 d}\right),\left(x_{11}, \ldots, x_{1 d}\right), \ldots,\left(x_{m 1}, \ldots, x_{m d}\right) ; f\right]
\end{aligned}
$$

\section{Theorem 2.1.}

The Lagrange multipolynomial $L\left(x ; f ; x_{0}, \ldots, x_{m}\right)$ is represented by the following Newton formula:

$$
\begin{aligned}
& L\left(x ; f ; x_{0}, \ldots, x_{m}\right)=L\left(\left(x_{1}, \ldots, x_{d}\right) ; f ;\left(x_{01}, \ldots x_{0 d}\right), \ldots,\left(x_{m 1}, \ldots, x_{m d}\right)\right) \\
& =\left[\left(x_{01}, \ldots x_{0 d}\right) ; f\right]+\left[\left(x_{01}, \ldots x_{0 d}\right),\left(x_{11}, \ldots x_{1 d}\right) ; f\right]\left(\left(x_{1}-x_{01}\right) \ldots\left(x_{d}-x_{0 d}\right)\right)+\ldots+ \\
& {\left[\left(x_{01}, \ldots x_{0 d}\right),\left(x_{11}, \ldots x_{1 d}\right), \ldots,\left(x_{m 1}, \ldots x_{m d}\right) ; f\right]\left(\left(x_{1}-x_{01}\right) \ldots\left(x_{d}-x_{0 d}\right)\right)\left(( x _ { 1 } - x _ { 1 1 } ) \ldots \left(x_{d}-\right.\right.} \\
& \left.\left.x_{1 d}\right)\right) \ldots\left(\left(x_{1}-x_{m 1-1}\right) \ldots\left(x_{d}-x_{m d-1}\right)\right)
\end{aligned}
$$

\section{Proof:}

For $m=1$, formula (10) follows from (3), (6) and (7) .

Assume that (10) is true for a number $m-1$.

By induction, let us prove that this formula is true for the number $m$, that is

$$
\begin{aligned}
& L\left(\left(x_{1}, \ldots, x_{d}\right) ; f ;\left(x_{01}, \ldots x_{0 d}\right), \ldots,\left(x_{m 1}, \ldots, x_{m d}\right)\right) \\
& =L\left(\left(x_{1}, \ldots, x_{d}\right) ; f ;\left(x_{01}, \ldots x_{0 d}\right), \ldots,\left(x_{m 1-1}, \ldots, x_{m d-1}\right)\right)+\left[\left(x_{01}, \ldots x_{0 d}\right), \ldots,\left(x_{m 1}, \ldots, x_{m d}\right) ; f\right] \\
& \left(\left(x_{1}-x_{01}\right) \ldots\left(x_{d}-x_{0 d}\right)\right) \ldots \quad\left(\left(x_{1}-x_{m 1-1}\right) \ldots\left(x_{d}-x_{m d-1}\right)\right) .
\end{aligned}
$$

Since both parts of this equality are multipolynomials of degree $\leq m$, it suffices to prove that this equality holds at all points $x_{i}, i=0, \ldots, m$.

By the definition of Lagrange multipolynomial (Definition1.1), for all $i=0, \ldots, m-1$, we have

$$
L\left(\left(x_{i 1}, \ldots, x_{i d}\right) ; f ;\left(x_{01}, \ldots x_{0 d}\right), \ldots,\left(x_{m 1-1}, \ldots, x_{m d-1}\right)\right)
$$


$+\left[\left(x_{01}, \ldots x_{0 d}\right), \ldots,\left(x_{m 1}, \ldots, x_{m d}\right) ; f\right]\left(\left(x_{i 1}-x_{01}\right) \ldots\left(x_{i d}-x_{0 d}\right)\right) \ldots\left(\left(x_{i 1}-x_{m 1-1}\right) \ldots\left(x_{i d}-\right.\right.$ $\left.\left.x_{m d-1}\right)\right)=f\left(\left(x_{i 1}, \ldots, x_{i d}\right)\right)+\mathrm{O}$

$=L\left(\left(x_{i 1}, \ldots, x_{i d}\right) ; f ;\left(x_{01}, \ldots x_{0 d}\right), \ldots,\left(x_{m 1}, \ldots, x_{m d}\right)\right)$,

for $i=m$ according to (9) we obtain

$L\left(\left(x_{m 1}, \ldots, x_{m d}\right) ; f ;\left(x_{01}, \ldots x_{0 d}\right), \ldots,\left(x_{m 1-1}, \ldots, x_{m d-1}\right)\right)+$

$\left[\left(x_{01}, \ldots x_{0 d}\right), \ldots,\left(x_{m 1}, \ldots, x_{m d}\right) ; f\right]\left(\left(x_{m 1}-x_{01}\right) \ldots\left(x_{m d}-x_{0 d}\right)\right) \ldots\left(\left(x_{m 1}-x_{m 1-1}\right) \ldots\left(x_{m d}-\right.\right.$ $\left.\left.x_{m d-1}\right)\right)$

$=f\left(\left(x_{m 1}, \ldots, x_{m d}\right)\right)=L\left(\left(x_{m 1}, \ldots, x_{m d}\right) ; f ;\left(x_{01}, \ldots x_{0 d}\right), \ldots,\left(x_{m 1}, \ldots, x_{m d}\right)\right)$

\section{Lemma 2.1.}

$L_{x_{j}}^{(m)}(f)=m ! \psi \quad\left[\left(x_{01}, \ldots x_{0 d}\right),\left(x_{11}, \ldots, x_{1 d}\right), \ldots,\left(x_{m 1}, \ldots, x_{m d}\right) ; f\right]$

where $\psi$ is a constant and $j=1, \ldots, d$.

\section{proof:}

We have

$$
\begin{aligned}
& \left(( ( x _ { 1 } - x _ { 0 1 } ) ( x _ { 1 } - x _ { 1 1 } ) \ldots ( x _ { 1 } - x _ { m 1 - 1 } ) ) ( ( x _ { 2 } - x _ { 0 2 } ) ( x _ { 2 } - x _ { 1 2 } ) \ldots ( x _ { 2 } - x _ { m 2 - 1 } ) ) \left(\left(x_{3}-\right.\right.\right. \\
& \left.\left.\left.x_{03}\right)\left(x_{3}-x_{13}\right) \ldots\left(x_{3}-x_{m 3-1}\right)\right) \ldots\left(\left(x_{d}-x_{0 d}\right)\left(x_{d}-x_{1 d}\right) \ldots\left(x_{d}-x_{m d-1}\right)\right)\right)^{(m)}
\end{aligned}
$$

$$
\begin{aligned}
& =\left(x_{j}^{m} \prod_{i=0}^{m-1} \prod_{\substack{\ell=1 \\
\ell \neq j}}^{d}\left(x_{\ell}-x_{i \ell}\right)\right)^{(m)}+\left(c_{1} x_{j}^{m-1} \prod_{i=0}^{m-1} \prod_{\substack{\ell=1 \\
\ell \neq j}}^{d}\left(x_{\ell}-x_{i \ell}\right)\right)^{(m)} \\
& +\cdots+\left(c_{m} \prod_{i=0}^{m-1} \prod_{\substack{\ell=1 \\
\ell \neq j}}^{d}\left(x_{\ell}-x_{i \ell}\right)\right)^{(m)}
\end{aligned}
$$


where $c_{1}, c_{2}, \ldots, c_{m}$ are constant and $j=1, \ldots, d$

$$
\begin{aligned}
& =m !\left(\prod_{i=0}^{m-1} \prod_{\substack{\ell=1 \\
\ell \neq j}}^{d}\left(x_{\ell}-x_{i \ell}\right)\right), \text { so } \\
& \begin{array}{c}
L_{x_{j}}^{(m)}(f)=m !\left(\prod_{i=0}^{m-1} \prod_{\substack{\ell=1 \\
\ell \neq j}}^{d}\left(x_{\ell}-x_{i \ell}\right)\right) \quad\left[\left(x_{01}, \ldots x_{0 d}\right),\left(x_{11}, \ldots, x_{1 d}\right), \ldots,\left(x_{m 1}, \ldots, x_{m d}\right) ; f\right] \\
=m ! \psi\left[\left(x_{01}, \ldots x_{0 d}\right),\left(x_{11}, \ldots, x_{1 d}\right), \ldots,\left(x_{m 1}, \ldots, x_{m d}\right) ; f\right]
\end{array}
\end{aligned}
$$

where $\psi=\prod_{i=0}^{m-1} \prod_{\substack{\ell=1 \\ \ell \neq j}}^{d}\left(x_{\ell}-x_{i \ell}\right)$ is a constant

\section{Lemma 2.2.}

The following identity is true

$$
\begin{aligned}
& \left(x_{0 j}-x_{m j}\right)\left[\left(x_{01}, \ldots x_{0 d}\right), \ldots,\left(x_{m 1}, \ldots, x_{m d}\right) ; f\right]=\left[\left(x_{01}, \ldots x_{0 d}\right), \ldots,\left(x_{m 1-1}, \ldots, x_{m d-1}\right) ; f\right] \\
& -\left[\left(x_{11}, \ldots x_{1 d}\right), \ldots,\left(x_{m 1}, \ldots, x_{m d}\right) ; f\right]
\end{aligned}
$$

where $j=1, \ldots, d$.

\section{Proof :}

Let $L\left(\left(x_{1}, \ldots, x_{d}\right)\right)=L\left(\left(x_{1}, \ldots, x_{d}\right) ; f ;\left(x_{01}, \ldots x_{0 d}\right), \ldots,\left(x_{m 1}, \ldots, x_{m d}\right)\right)$.

It follows from (10) and (11) that

$$
\begin{aligned}
& L_{x_{j}}^{(m-1)}(f)=\left[\left(x_{01}, \ldots x_{0 d}\right), \ldots,\left(x_{m 1-1}, \ldots, x_{m d-1}\right) ; f\right](m-1) ! \psi+ \\
& {\left[\left(x_{01}, \ldots x_{0 d}\right), \ldots,\left(x_{m 1}, \ldots, x_{m d}\right) ; f\right] \psi\left(m ! x_{j}-(m-1) !\left(x_{0 j}+\cdots+x_{m j-1}\right)\right) .}
\end{aligned}
$$

Interchanging the points $x_{0}=\left(x_{01}, \ldots x_{0 d}\right)$ and $x_{m}=\left(x_{m 1}, \ldots, x_{m d}\right)$ in (10) we get

$$
\begin{aligned}
& L_{x_{j}}^{(m-1)}(f)=\left[\left(x_{m 1}, \ldots x_{m d}\right),\left(x_{11}, \ldots, x_{1 d}\right), \ldots,\left(x_{m 1-1}, \ldots, x_{m d-1}\right) ; f\right](m-1) ! \psi \\
& +\left[\left(x_{m 1}, \ldots, x_{m d}\right),\left(x_{11}, \ldots, x_{1 d}\right), \ldots,\left(x_{m 1-1}, \ldots, x_{m d-1}\right),\left(x_{01}, \ldots, x_{0 d}\right) ; f\right] \\
& \psi\left(m ! x_{j}-(m-1) !\left(x_{m j}+x_{1 j}+\ldots+x_{m j-1}\right)\right) \\
& =\left[\left(x_{11}, \ldots x_{1 d}\right), \ldots,\left(x_{m 1}, \ldots, x_{m d}\right) ; f\right](m-1) ! \psi
\end{aligned}
$$


$+\left[\left(x_{01}, \ldots, x_{0 d}\right), \ldots,\left(x_{m 1}, \ldots, x_{m d}\right) ; f\right] \psi\left(m ! x_{j}-(m-1) !\left(x_{1 j}+\cdots+x_{m j}\right)\right)$.

\section{Subtracting equalities}

$\left[\left(x_{01}, \ldots x_{0 d}\right), \ldots,\left(x_{m 1-1}, \ldots, x_{m d-1}\right) ; f\right](m-1) ! \psi+\left[\left(x_{01}, \ldots x_{0 d}\right), \ldots,\left(x_{m 1}, \ldots, x_{m d}\right) ; f\right] \psi$ $\left(m ! x_{j}-(m-1) !\left(x_{0 j}+\cdots+x_{m j-1}\right)\right)$, and

$\left[\left(x_{11}, \ldots x_{1 d}\right), \ldots,\left(x_{m 1}, \ldots, x_{m d}\right) ; f\right](m-1) ! \psi+\left[\left(x_{01}, \ldots x_{0 d}\right), \ldots,\left(x_{m 1}, \ldots x_{m d}\right) ; f\right] \psi\left(m ! x_{j}-\right.$ $\left.(m-1) !\left(x_{1 j}+\cdots+x_{m j}\right)\right)$, we get

$\left(\left[\left(x_{01}, \ldots x_{0 d}\right), \ldots,\left(x_{m 1-1}, \ldots, x_{m d-1}\right) ; f\right]-\left[\left(x_{11}, \ldots x_{1 d}\right), \ldots,\left(x_{m 1}, \ldots, x_{m d}\right) ; f\right]\right)(m-1) ! \psi$

$-\left((m-1) ! \psi\left[\left(x_{01}, \ldots x_{0 d}\right), \ldots,\left(x_{m 1}, \ldots, x_{m d}\right) ; f\right]\left(x_{0 j}-x_{m j}\right)\right)=0$

By dividing on $(m-1) ! \psi$, we get

$$
\begin{aligned}
& \left(x_{0 j}-x_{m j}\right)\left[\left(x_{01}, \ldots x_{0 d}\right), \ldots,\left(x_{m 1}, \ldots, x_{m d}\right) ; f\right] \\
& =\left[\left(x_{01}, \ldots x_{0 d}\right), \ldots,\left(x_{m 1-1}, \ldots, x_{m d-1}\right) ; f\right]-\left[\left(x_{11}, \ldots x_{1 d}\right), \ldots,\left(x_{m 1}, \ldots, x_{m d}\right) ; f\right]
\end{aligned}
$$

Now let $x_{0}, x_{1} \in[a, b]^{d}$ and let a function $f$ be absolutely continuous on $[a, b]^{d}$. Then according to the Lebesgue theorem we have

$$
\begin{aligned}
& f\left(\left(x_{11}, \ldots x_{1 d}\right)\right)-f\left(\left(x_{01}, \ldots x_{0 d}\right)\right) \\
& =\int_{x_{01}}^{x_{11}} \ldots \int_{x_{0 d}}^{x_{1 d}} f_{t_{1}, t_{2}, \ldots, t_{d}}\left(\left(t_{1}, \ldots, t_{d}\right)\right) d t_{1} \ldots d t_{d} .
\end{aligned}
$$

Performing the change of variables $t_{1}=x_{01}+\left(x_{11}-x_{01}\right) \tilde{t}_{1}, \ldots, t_{d}=x_{0 d}+\left(x_{1 d}-x_{0 d}\right) \tilde{t}_{1}$ we obtain

$$
\begin{aligned}
& {\left[\left(x_{01}, \ldots x_{0 d}\right),\left(x_{11}, \ldots x_{1 d}\right) ; f\right]} \\
& =\frac{1}{\left(x_{11}-x_{01}\right) \ldots\left(x_{1 d}-x_{0 d}\right)} \int_{x_{01}}^{x_{11}} \ldots \int_{x_{0 d}}^{x_{1 d}} f_{t_{1}, \ldots, t_{d}}\left(\left(t_{1}, \ldots, t_{d}\right)\right) d t_{1} \ldots d t_{d} \\
& =\int_{0}^{1} \ldots \int_{0}^{1} f^{\prime}\left(\left(x_{01}+\left(x_{11}-x_{01}\right) \tilde{t}_{1}, \ldots, x_{0 d}+\left(x_{1 d}-x_{0 d}\right) \tilde{t}_{1}\right)\right) d \tilde{t}_{1}^{d}
\end{aligned}
$$

where $d t_{1}=\left(x_{11}-x_{01}\right) d \tilde{t}_{1}, \ldots, d t_{d}=\left(x_{1 d}-x_{0 d}\right) d \tilde{t}_{1}, \quad f^{\prime}=f_{t_{1}, \ldots, t_{d}} \quad$ and $d \tilde{t}_{1}^{d}=d \tilde{t}_{1} d \tilde{t}_{1} \ldots d \tilde{t}_{1}, d$ times . 
A similar representation is true for any $m$ by virtue of the following theorem:

\section{Theorem 2.2.}

Let $x_{i} \in[a, b]^{d}$ where $x_{i}=\left(x_{i 1}, \ldots, x_{i d}\right)$ for $i=0, \ldots, m$.

If the function $f$ has the absolute continuous $(m-1)$ th derivative on $[a, b]^{d}$, then

$$
\begin{aligned}
& {\left[\left(x_{01}, \ldots x_{0 d}\right),\left(x_{11}, \ldots x_{1 d}\right), \ldots,\left(x_{m 1}, \ldots, x_{m d}\right) ; f\right]} \\
& \begin{aligned}
=\int_{0}^{1} \ldots \int_{0}^{1} \int_{0}^{\tilde{t}_{1}} & \ldots \int_{0}^{\tilde{t}_{1}} \ldots \int_{0}^{\tilde{t}_{m-1}} \ldots \int_{0}^{\tilde{t}_{m-1}} f^{(m)}\left(\left(x_{01}, \ldots x_{0 d}\right)\right. \\
& +\left(\left(x_{11}-x_{01}\right) \tilde{t}_{1}, \ldots,\left(x_{1 d}-x_{0 d}\right) \tilde{t}_{1}\right)+\ldots \\
& \left.+\left(\left(x_{m 1}-x_{m 1-1}\right) \tilde{t}_{m}, \ldots,\left(x_{m d}-x_{m d-1}\right) \tilde{t}_{m}\right)\right) d \tilde{t}_{m}^{d} \ldots d \tilde{t}_{1}^{d}
\end{aligned}
\end{aligned}
$$

\section{Proof:}

Assume that representation (14) is true for a number $m-1$. By induction, let us prove that (14) is also true for the number $m$. Denote $\tilde{t}_{0}:=1$. According to relation (12) and the induction hypothesis, we have

$$
\begin{aligned}
& \left(x_{m j}-x_{m j-1}\right)\left[\left(x_{01}, \ldots x_{0 d}\right), \ldots,\left(x_{m 1}, \ldots, x_{m d}\right) ; f\right] \\
& =\left[\left(x_{01}, \ldots x_{0 d}\right), \ldots,\left(x_{m 1-2}, \ldots, x_{m d-2}\right),\left(x_{m 1}, \ldots x_{m d}\right) ; f\right] \\
& \quad-\left[\left(x_{01}, \ldots, x_{0 d}\right), \ldots,\left(x_{m 1-1}, \ldots, x_{m d-1}\right) ; f\right]
\end{aligned}
$$

where

$$
\begin{aligned}
& f^{(m)}=f_{\underbrace{}_{\text {times }}}^{t_{1} \ldots t_{1}, t_{2} \ldots t_{2}, \ldots, \underbrace{t_{d} \ldots t_{d}}_{m \text { times }}}, \\
& v_{1}=x_{01}+\cdots+\left(x_{m 1-2}-x_{m 1-3}\right) \tilde{t}_{m-2}+\left(x_{m 1}-x_{m 1-2}\right) \tilde{t}_{m-1}, \\
& \quad \vdots \\
& v_{d}=x_{0 d}+\cdots+\left(x_{m d-2}-x_{m d-3}\right) \tilde{t}_{m-2}+\left(x_{m d}-x_{m d-2}\right) \tilde{t}_{m-1},
\end{aligned}
$$




$$
\begin{gathered}
u_{1}=x_{01}+\cdots+\left(x_{m 1-1}-x_{m 1-2}\right) \tilde{t}_{m-1}, \\
\vdots \\
u_{d}=x_{0 d}+\cdots+\left(x_{m d-1}-x_{m d-2}\right) \tilde{t}_{m-1} .
\end{gathered}
$$

It remains to introduce a new integration variable $\tilde{t}_{m}$ instead of $t=\left(t_{1}, \ldots, t_{d}\right)$ in the last integral by using the change of variables

$$
\begin{aligned}
& t_{1}=x_{01}+\left(x_{11}-x_{01}\right) \tilde{t}_{1}+\cdots+\left(x_{m 1-1}-x_{m 1-2}\right) \tilde{t}_{m-1}+\left(x_{m 1}-x_{m 1-1}\right) \tilde{t}_{m}, \\
& \quad \vdots \\
& t_{d}=x_{0 d}+\left(x_{1 d}-x_{0 d}\right) \tilde{t}_{1}+\cdots+\left(x_{m d-1}-x_{m d-2}\right) \tilde{t}_{m-1}+\left(x_{m d}-x_{m d-1}\right) \tilde{t}_{m} .
\end{aligned}
$$

And then note that this change of variables transforms the segment $\left[0, \tilde{t}_{m}\right]^{d}$ into the segment that connects the points $u=\left(u_{1}, \ldots, u_{d}\right)$ and $v=\left(v_{1}, \ldots, v_{d}\right)$

\section{Lemma 2.3.}

Let $i \in N, i \leq m$ and let $x_{i} \in[a, b]^{d}$ then

$$
\left[\left(x_{01}, \ldots, x_{0 d}\right), \ldots,\left(x_{m 1}, \ldots, x_{m d}\right) ; f\right]=\left[\left(x_{i 1}, \ldots, x_{i d}\right), \ldots,\left(x_{m 1}, \ldots, x_{m d}\right) ; f_{i}\right]
$$

where $f_{i}\left(\left(x_{1}, \ldots, x_{d}\right)\right)=\left[\left(x_{01}, \ldots, x_{0 d}\right), \ldots,\left(x_{i 1-1}, \ldots, x_{i d-1}\right) ;\left(x_{1}, \ldots, x_{d}\right) ; f\right]$

\section{proof :}

Can easily be proved by induction with the use of (12)

\section{Lemma 2.4.}

Let $k \in N, k \leq m$, and let $x_{i} \in[a, b]^{d}$ for all $i=0, \ldots, m$. If a function $f$ is $k$ times continuously differentiable on $[a, b]^{d}$ or $f$ has the $(k-1)$ th absolutely continuous derivative on $[a, b]^{d}$, then

$$
\left[\left(x_{01}, \ldots, x_{0 d}\right), \ldots,\left(x_{m 1}, \ldots, x_{m d}\right) ; f\right]=\int_{0}^{1} \ldots \int_{0}^{1}\left[\left(x_{11}, \ldots, x_{1 d}\right), \ldots,\left(x_{m 1}, \ldots, x_{m d}\right) ; f_{\tilde{t}_{1}^{d}}\right] d \tilde{t}_{1}^{d}
$$

\section{Proof:}

From (13), (14) and (15) we get 


$$
\begin{aligned}
& {\left[\left(x_{01}, \ldots, x_{0 d}\right), \ldots,\left(x_{m 1}, \ldots, x_{m d}\right) ; f\right]} \\
& =\int_{0}^{1} \ldots \int_{0}^{1} \ldots \int_{0}^{\tilde{t}_{k-1}} \ldots \int_{0}^{\tilde{t}_{k-1}}\left[\left(x_{k 1}, \ldots x_{k d}\right), \ldots,\left(x_{m 1}, \ldots, x_{m d}\right) ; f_{\tilde{t}_{1}^{d}, \ldots, \tilde{t}_{k}^{d}}\right] d \tilde{t}_{k}^{d} \ldots d \tilde{t}_{1}^{d},
\end{aligned}
$$

where

$$
\begin{aligned}
& f_{\tilde{t}_{1}^{d}, \ldots, \tilde{t}_{k}^{d}}\left(\left(x_{1}, \ldots x_{d}\right)\right) \\
& =f^{(k)}\left(\left(x_{01}, \ldots, x_{0 d}\right)+\left(\left(x_{11}, \ldots, x_{1 d}\right)-\left(x_{01}, \ldots, x_{0 d}\right)\right) \tilde{t}_{1}+\ldots\right. \\
& \quad+\left(\left(x_{k 1-1}, \ldots, x_{k d-1}\right)-\left(x_{k 1-2}, \ldots, x_{k d-2}\right)\right) \tilde{t}_{k-1} \\
& \left.\quad+\left(\left(x_{k 1}, \ldots, x_{k d}\right)-\left(x_{k 1-1}, \ldots, x_{k d-1}\right)\right) \tilde{t}_{k}\right)
\end{aligned}
$$

In particular , if $k=1$, then

$$
\begin{aligned}
& {\left[\left(x_{01}, \ldots, x_{0 d}\right), \ldots,\left(x_{m 1}, \ldots, x_{m d}\right) ; f\right]} \\
& =\int_{0}^{1} \ldots \int_{0}^{1}\left[\left(x_{11}, \ldots, x_{1 d}\right), \ldots,\left(x_{m 1}, \ldots, x_{m d}\right) ; f_{\tilde{t}_{1}^{d}}\right] d \tilde{t}_{1}^{d}
\end{aligned}
$$

\section{Finite differences}

In this section, we assume that the points $x_{i}=\left(x_{i 1}, \ldots, x_{i d}\right)$ are equidistant, that is , for all $i=0, \ldots, m$ we have

$x_{i 1}=x_{01}+i h_{1}, \ldots, x_{i d}=x_{0 d}+i h_{d}, h \in R^{d}, h_{j} \neq 0, j=1, \ldots, d$.

For the Lagrange interpolation multipolynomial

$$
\begin{aligned}
& L\left(\left(x_{1}, \ldots, x_{d}\right)\right)=L\left(\left(x_{1}, \ldots, x_{d}\right) ; f ;\left(x_{01}, \ldots, x_{0 d}\right), \ldots,\left(x_{m 1-1}, \ldots, x_{m d-1}\right)\right) \\
& =\sum_{k=0}^{m-1} f\left(\left(x_{k 1}, \ldots, x_{k d}\right)\right) I_{k}\left(\left(x_{1}, \ldots, x_{d}\right),\left(x_{01}, \ldots, x_{0 d}\right), \ldots,\left(x_{m 1-1}, \ldots, x_{m d-1}\right)\right) .
\end{aligned}
$$

We determine the values of the new version of the fundamental Lagrange multipolynomials $I_{k}$ at the point $x_{1}=x_{m 1}, \ldots, x_{d}=x_{m d}$.

According to (4), we have 


$$
\begin{aligned}
& I_{k}\left(\left(x_{m 1}, \ldots, x_{m d}\right),\left(x_{01}, \ldots, x_{0 d}\right), \ldots,\left(x_{m 1-1}, \ldots, x_{m d-1}\right)\right) \\
& =\prod_{\substack{i=0 \\
k \neq i}}^{m-1} \frac{\left(x_{m 1}-x_{i 1}\right) \ldots\left(x_{m d}-x_{i d}\right)}{\left(x_{k 1}-x_{i 1}\right) \ldots\left(x_{k d}-x_{i d}\right)} \\
& =\prod_{\substack{i=0 \\
i \neq k}}^{m-1} \frac{\left(x_{01}+m h_{1}-x_{01}-i h_{1}\right) \ldots\left(x_{0 d}+m h_{d}-x_{0 d}-i h_{d}\right)}{\left(x_{01}+k h_{1}-x_{01}-i h_{1}\right) \ldots\left(x_{0 d}+k h_{d}-x_{0 d}-i h_{d}\right)} \\
& =\prod_{\substack{i=0 \\
i \neq k}}^{m-1} \frac{(m-i) h_{1} \ldots(m-i) h_{d}}{(k-i) h_{1} \ldots(k-i) h_{d}} \\
& =\prod_{\substack{i=0 \\
i \neq k}}^{m-1} \frac{(m-i) \ldots(m-i)}{(k-i) \ldots(k-i)} \\
& =\left(-(-1)^{m-k}\left(\begin{array}{c}
m \\
k
\end{array}\right)\right) \ldots\left(-(-1)^{m-k}\left(\begin{array}{c}
m \\
k
\end{array}\right)\right) \\
& =\left(-(-1)^{m-k}\left(\begin{array}{c}
m \\
k
\end{array}\right)\right)^{d}
\end{aligned}
$$

We represent the difference $f\left(\left(x_{m 1}, \ldots, x_{m d}\right)\right)-L\left(\left(x_{m 1}, \ldots, x_{m d}\right)\right)$ in the form

$$
\begin{aligned}
& f\left(\left(x_{m 1}, \ldots, x_{m d}\right)\right)-L\left(\left(x_{m 1}, \ldots, x_{m d}\right)\right) \\
& =\sum_{k=0}^{m}\left((-1)^{m-k}\left(\begin{array}{c}
m \\
k
\end{array}\right)\right)^{d} f\left(\left(x_{01}+k h_{1}, \ldots, x_{0 d}+k h_{d}\right)\right) .
\end{aligned}
$$

\section{Lemma 3.1.}

$$
\begin{aligned}
& \Delta_{h}^{m}\left(f ;\left(x_{01}, \ldots, x_{0 d}\right)\right) \\
& =(m !)^{d}\left(h_{1} \ldots h_{d}\right)^{m}\left[\left(x_{01}, \ldots, x_{0 d}\right),\left(x_{01}+h_{1}, \ldots, x_{0 d}+h_{d}\right), \ldots,\left(x_{01}+m h_{1}, \ldots, x_{0 d}+m h_{d}\right) ; f\right]
\end{aligned}
$$

\section{Proof:}


Since $f\left(\left(x_{m 1}, \ldots, x_{m d}\right)\right)-L\left(\left(x_{m 1}, \ldots, x_{m d}\right)\right)$

$=\sum_{k=0}^{m}\left((-1)^{m-k}\left(\begin{array}{c}m \\ k\end{array}\right)\right)^{d} f\left(\left(x_{01}+k h_{1}, \ldots, x_{0 d}+k h_{d}\right)\right)$

and

$f\left(\left(x_{m 1}, \ldots, x_{m d}\right)\right)-L\left(\left(x_{m 1}, \ldots, x_{m d}\right) ; f ;\left(x_{01}, \ldots, x_{0 d}\right), \ldots,\left(x_{m 1-1}, \ldots, x_{m d-1}\right)\right)$

$=\prod_{k=0}^{m-1}\left(\left(x_{m 1-} x_{k 1}\right) \ldots\left(x_{m d}-x_{k d}\right)\right)\left[\left(x_{01}, \ldots, x_{0 d}\right),\left(x_{11}, \ldots, x_{1 d}\right), \ldots,\left(x_{m 1}, \ldots, x_{m d}\right) ; f\right]$,

then

$\Delta_{h}^{m}\left(f ;\left(x_{01}, \ldots, x_{0 d}\right)\right)$

$=\prod_{k=0}^{m-1}\left(\left(x_{01}+m h_{1}-x_{01}-k h_{1}\right) \ldots\left(x_{0 d}+m h_{d}-x_{0 d}-k h_{d}\right)\right)\left[\left(x_{01}, \ldots x_{0 d}\right),\left(x_{01}+h_{1}, \ldots, x_{0 d}\right.\right.$ $\left.\left.+h_{d}\right), \ldots,\left(x_{01}+m h_{1}, \ldots, x_{0 d}+m h_{d}\right) ; f\right]$

$=\prod_{k=0}^{m-1}\left(\left((m-k) h_{1}\right) \ldots\left((m-k) h_{d}\right)\right)\left[\left(x_{01}, \ldots, x_{0 d}\right),\left(x_{01}+h_{1}, \ldots, x_{0 d}+h_{d}\right), \ldots,\left(x_{01}\right.\right.$ $\left.\left.+m h_{1}, \ldots, x_{0 d}+m h_{d}\right) ; f\right]$

$=\left(\left(m h_{1}\right)\left((m-1) h_{1}\right)\left((m-2) h_{1}\right) \ldots\left((m-m+1) h_{1}\right)\right) \ldots\left(\left(m h_{d}\right)\left((m-1) h_{d}\right)((m\right.$ $\left.\left.-2) h_{d}\right) \ldots\left((m-m+1) h_{d}\right)\right)\left[\left(x_{01}, \ldots, x_{0 d}\right),\left(x_{01}+h_{1}, \ldots, x_{0 d}+h_{d}\right), \ldots,\left(x_{01}\right.\right.$ $\left.\left.+m h_{1}, \ldots, x_{0 d}+m h_{d}\right) ; f\right]$

$=\left(m ! h_{1}{ }^{m}\right) \ldots\left(m ! h_{d}^{m}\right)\left[\left(x_{01}, \ldots, x_{0 d}\right),\left(x_{01}+h_{1}, \ldots, x_{0 d}+h_{d}\right), \ldots,\left(x_{01}+m h_{1}, \ldots, x_{0 d}\right.\right.$ $\left.\left.+m h_{d}\right) ; f\right]$

$=(m !)^{d}\left(h_{1} \ldots h_{d}\right)^{m}\left[\left(x_{01}, \ldots, x_{0 d}\right),\left(x_{01}+h_{1}, \ldots, x_{0 d}+h_{d}\right), \ldots,\left(x_{01}+m h_{1}, \ldots, x_{0 d}+m h_{d}\right) ; f\right]$

\section{Lemma 3.2.}

Let $x_{0} \in[a, b]^{d}, h_{j}>0, j=1, \ldots, d, x_{k}=\left(x_{k 1}, \ldots, x_{k d}\right)$ such that

$x_{k 1}=x_{01}+k h_{1}, \ldots, x_{k d}=x_{0 d}+k h_{d}$ and $x_{m} \in[a, b]^{d}, x_{m}=\left(x_{m 1}, \ldots, x_{m d}\right)$. 
If $F \in L_{p}^{1}\left([a, b]^{d}\right)$, then for every $x \in[a, b]^{d}$ the following inequality is true:

$$
\begin{aligned}
& \left\|F\left(\left(x_{1}, \ldots, x_{d}\right)\right)-L\left(\left(x_{1}, \ldots, x_{d}\right) ; F ;\left(x_{01}, \ldots, x_{0 d}\right), \ldots,\left(x_{m 1}, \ldots, x_{m d}\right)\right)\right\|_{L_{p}[a, b]^{d}} \\
& \leq C(p) \frac{1}{(m !)^{d}\left(h_{1} \ldots h_{d}\right)^{m}} \|\left(\left(x_{1}-x_{01}\right) \ldots\left(x_{d}-x_{0 d}\right)\right) \ldots\left(( x _ { 1 } - x _ { m 1 } ) \ldots \left(x_{d}\right.\right. \\
& \left.\left.-x_{m d}\right)\right) \|_{L_{p}[a, b]^{d}} \omega_{m}\left(F^{\prime}, h,[a, b]^{d}\right)_{p}
\end{aligned}
$$

\section{Proof:}

For every $\tilde{t}_{1} \in[0,1]$, we set

$$
\begin{aligned}
F_{\tilde{t}_{1}^{d}}\left(\left(u_{1}, \ldots, u_{d}\right)\right) & =F^{\prime}\left(\left(x_{1}, \ldots, x_{d}\right)+\left(\left(u_{1}, \ldots, u_{d}\right)-\left(x_{1}, \ldots, x_{d}\right)\right) \tilde{t}_{1}\right) \\
& =F^{\prime}\left(\left(x_{1}+\left(u_{1}-x_{1}\right) \tilde{t}_{1}, \ldots, x_{d}+\left(u_{d}-x_{d}\right) \tilde{t}_{1}\right)\right)
\end{aligned}
$$

where $u \in[a, b]^{d}$.

Then relations (16) and (18) yield

$$
\begin{aligned}
& {\left[\left(x_{1}, \ldots, x_{d}\right),\left(x_{01}, \ldots, x_{0 d}\right), \ldots,\left(x_{m 1}, \ldots, x_{m d}\right) ; F\right]} \\
& =\int_{0}^{1} \ldots \int_{0}^{1}\left[\left(x_{01}, \ldots, x_{0 d}\right), \ldots,\left(x_{m 1}, \ldots, x_{m d}\right) ; F_{t_{1}^{d}}\right] d \tilde{t}_{1}{ }^{d} \\
& =\frac{1}{(m !)^{d}\left(h_{1} \ldots h_{d}\right)^{m}} \int_{0}^{1} \ldots \int_{0}^{1} \Delta_{h}^{m}\left(F_{\tilde{t}_{1}^{d}} ;\left(x_{01}, \ldots, x_{0 d}\right)\right) d \tilde{t}_{1}{ }^{d}
\end{aligned}
$$

Since

$$
\begin{aligned}
& \left\|\Delta_{h}^{m}\left(F_{\tilde{t}_{1}^{d}} ;\left(u_{1}, \ldots, u_{d}\right)\right)\right\|_{L_{p}[a, b]^{d}} \\
& =\left\|\Delta_{h \tilde{t}_{1}}^{m}\left(F^{\prime} ;\left(x_{1}+\left(u_{1}-x_{1}\right) \tilde{t}_{1}, \ldots, x_{d}+\left(u_{d}-x_{d}\right) \tilde{t}_{1}\right)\right)\right\|_{L_{p}[a, b]^{d}} \\
& \leq \omega_{m}\left(F^{\prime}, h \tilde{t}_{1},[a, b]^{d}\right)_{p} \leq \omega_{m}\left(F^{\prime}, h,[a, b]^{d}\right)_{p} \\
& \text { then }
\end{aligned}
$$$$
\left\|\left[\left(x_{1}, \ldots, x_{d}\right),\left(x_{01}, \ldots, x_{0 d}\right), \ldots,\left(x_{m 1}, \ldots, x_{m d}\right) ; F\right]\right\|_{L_{p}[a, b]^{d}}
$$ 
$=\left\|\frac{1}{(m !)^{d}\left(h_{1} \ldots h_{d}\right)^{m}} \int_{0}^{1} \ldots \int_{0}^{1} \Delta_{h}^{m}\left(F_{\tilde{t}_{1}^{d}} ;\left(x_{01}, \ldots, x_{0 d}\right)\right) d \tilde{t}_{1}^{d}\right\|_{L_{p}[a, b]^{d}}$

$\leq C(p) \frac{1}{(m !)^{d}\left(h_{1} \ldots h_{d}\right)^{m}}\left\|\Delta_{h}^{m}\left(F_{\tilde{t}_{1}^{d}} ;\left(x_{01}, \ldots, x_{0 d}\right)\right)\right\|_{L_{p}[a, b]^{d}}$

$=C(p) \frac{1}{(m !)^{d}\left(h_{1} \ldots h_{d}\right)^{m}}\left\|\Delta_{h \tilde{t}_{1}}^{m}\left(F^{\prime} ;\left(x_{1}+\left(x_{01}-x_{1}\right) \tilde{t}_{1}, \ldots, x_{d}+\left(x_{0 d}-x_{d}\right) \tilde{t}_{1}\right)\right)\right\|_{L_{p}[a, b]^{d}}$

$\leq C(p) \frac{1}{(m !)^{d}\left(h_{1} \ldots h_{d}\right)^{m}} \omega_{m}\left(F^{\prime}, h,[a, b]^{d}\right)_{p}$,

relation (19) follows from (9) such that

$F\left(\left(x_{1}, \ldots, x_{d}\right)\right)-L\left(\left(x_{1}, \ldots, x_{d}\right) ; F ;\left(x_{01}, \ldots, x_{0 d}\right), \ldots,\left(x_{m 1}, \ldots, x_{m d}\right)\right)$

$=\left(\left(x_{1}-x_{01}\right) \ldots\left(x_{d}-x_{0 d}\right)\right) \ldots\left(\left(x_{1}-x_{m 1}\right) \ldots\left(x_{d}\right.\right.$

$\left.\left.-x_{m d}\right)\right)\left[\left(x_{1}, \ldots, x_{d}\right),\left(x_{01}, \ldots, x_{0 d}\right), \ldots,\left(x_{m 1}, \ldots, x_{m d}\right) ; F\right]$,

since

$\left\|\left[\left(x_{1}, \ldots, x_{d}\right),\left(x_{01}, \ldots, x_{0 d}\right), \ldots,\left(x_{m 1}, \ldots, x_{m d}\right) ; F\right]\right\|_{L_{p}[a, b]^{d}}$

$\leq C(p) \frac{1}{(m !)^{d}\left(h_{1} \ldots h_{d}\right)^{m}} \omega_{m}\left(F^{\prime}, h,[a, b]^{d}\right)_{p}$,

then

$\left\|F\left(\left(x_{1}, \ldots, x_{d}\right)\right)-L\left(\left(x_{1}, \ldots, x_{d}\right) ; F ;\left(x_{01}, \ldots, x_{0 d}\right), \ldots,\left(x_{m 1}, \ldots, x_{m d}\right)\right)\right\|_{L_{p}[a, b]^{d}}$

$\leq C(p) \frac{1}{(m !)^{d}\left(h_{1} \ldots h_{d}\right)^{m}} \|\left(\left(x_{1}-x_{01}\right) \ldots\left(x_{d}-x_{0 d}\right)\right) \ldots\left(\left(x_{1}-x_{m 1}\right) \ldots\left(x_{d}\right.\right.$ $\left.\left.-x_{m d}\right)\right) \|_{L_{p}[a, b]^{d}} \omega_{m}\left(F^{\prime}, h,[a, b]^{d}\right)_{p}$

\section{Proof of theorem 1.1.}

Let $x_{k}=\left(x_{k 1}, \ldots, x_{k d}\right)$ and $x_{0}=\left(x_{01}, \ldots, x_{0 d}\right)$ such that $x_{0 j}:=a$, $h_{j}:=\frac{b-a}{m}, j=1, \ldots, d$ and $x_{k 1}=x_{01}+k h_{1}, \ldots, x_{k d}=x_{0 d}+k h_{d}$, 


$$
\begin{aligned}
& F\left(\left(x_{1}, \ldots, x_{d}\right)\right):=\int_{a}^{x_{1}} \ldots \int_{a}^{x_{d}} f\left(\left(u_{1}, \ldots, u_{d}\right)\right) d u_{1} \ldots d u_{d} \\
& G\left(\left(x_{1}, \ldots, x_{d}\right)\right):=F\left(\left(x_{1}, \ldots, x_{d}\right)\right)-L\left(\left(x_{1}, \ldots, x_{d}\right) ; F ;\left(x_{01}, \ldots, x_{0 d}\right), \ldots,\left(x_{m 1}, \ldots, x_{m d}\right)\right), \\
& g\left(\left(x_{1}, \ldots, x_{d}\right)\right)=G^{\prime}\left(\left(x_{1}, \ldots, x_{d}\right)\right), \\
& \omega_{m}\left(\left(t_{1}, \ldots, t_{d}\right)\right)=\omega_{m}\left(\left(t_{1}, \ldots, t_{d}\right), f,[a, b]^{d}\right)_{p} \equiv \omega_{m}\left(\left(t_{1}, \ldots, t_{d}\right), g,[a, b]^{d}\right)_{p} .
\end{aligned}
$$

We fix $x \in[a, b]^{d}$, choose $\delta$ for which $(x+m \delta) \in[a, b]^{d}$ and let $\delta^{\prime}=\left(t_{1} \delta_{1}, \ldots, t_{d} \delta_{d}\right)$. As a result, we get

$$
\begin{aligned}
& \begin{array}{l}
\int_{0}^{1} \ldots \int_{0}^{1} \Delta_{\delta^{\prime}}^{m}\left(g ;\left(x_{1}, \ldots, x_{d}\right)\right) d t_{1} \ldots d t_{d} \\
=(-1)^{m d} g\left(\left(x_{1}, \ldots, x_{d}\right)\right) \\
\quad+\sum_{k=1}^{m}\left((-1)^{m-k}\left(\begin{array}{c}
m \\
k
\end{array}\right)\right)^{d} \int_{0}^{1} \ldots \int_{0}^{1} g\left(\left(x_{1}+k t_{1} \delta_{1}, \ldots, x_{d}+k t_{d} \delta_{d}\right)\right) d t_{1} \ldots d t_{d}
\end{array} \\
& \begin{aligned}
=(-1)^{m d} g\left(\left(x_{1}, \ldots, x_{d}\right)\right) \\
\quad+\sum_{k=1}^{m}\left((-1)^{m-k}\left(\begin{array}{c}
m \\
k
\end{array}\right)\right)^{d} \int_{0}^{1} \ldots \int_{0}^{1} G^{\prime}\left(\left(x_{1}+k t_{1} \delta_{1}, \ldots, x_{d}+k t_{d} \delta_{d}\right)\right) d t_{1} \ldots d t_{d} \\
=(-1)^{m d} g\left(\left(x_{1}, \ldots, x_{d}\right)\right) \\
\quad+\sum_{k=1}^{m}\left((-1)^{m-k}\left(\begin{array}{c}
m \\
k
\end{array}\right)\right)^{d} \frac{1}{k^{d}\left(\delta_{1} \ldots \delta_{d}\right)}\left(G\left(\left(x_{1}+k \delta_{1}, \ldots, x_{d}+k \delta_{d}\right)\right)\right. \\
\left.\quad-G\left(\left(x_{1}, \ldots, x_{d}\right)\right)\right),
\end{aligned}
\end{aligned}
$$

whence

$$
\begin{aligned}
\left|g\left(\left(x_{1}, \ldots, x_{d}\right)\right)\right| & \\
& \leq \int_{0}^{1} \ldots \int_{0}^{1}\left|\Delta_{\delta^{\prime}}^{m}\left(g ;\left(x_{1}, \ldots, x_{d}\right)\right)\right| d t_{1} \ldots d t_{d} \\
& +\mid \sum_{k=1}^{m}\left((-1)^{m-k}\left(\begin{array}{c}
m \\
k
\end{array}\right)\right)^{d} \frac{1}{k^{d}\left(\delta_{1} \ldots \delta_{d}\right)}\left(G\left(\left(x_{1}+k \delta_{1}, \ldots, x_{d}+k \delta_{d}\right)\right)\right. \\
& \left.-G\left(\left(x_{1}, \ldots, x_{d}\right)\right)\right) \mid
\end{aligned}
$$


Then

$$
\begin{aligned}
& \|g\|_{L_{p}[a, b]^{d}} \leq \| \int_{0}^{1} \ldots \int_{0}^{1}\left|\Delta_{\delta^{\prime}}^{m}\left(g ;\left(x_{1}, \ldots, x_{d}\right)\right)\right| d t_{1} \ldots d t_{d} \\
& +\sum_{k=1}^{m}\left((-1)^{m-k}\left(\begin{array}{c}
m \\
k
\end{array}\right)\right)^{d} \frac{1}{k^{d}\left(\delta_{1} \ldots \delta_{d}\right)}\left(G\left(\left(x_{1}+k \delta_{1}, \ldots, x_{d}+k \delta_{d}\right)\right)\right. \\
& \left.-G\left(\left(x_{1}, \ldots, x_{d}\right)\right)\right) \|_{L_{p}[a, b]^{d}} \\
& \leq C(p)\left\|\int_{0}^{1} \ldots \int_{0}^{1} \Delta_{\delta^{\prime}}^{m}\left(g ;\left(x_{1}, \ldots, x_{d}\right)\right) d t_{1} \ldots d t_{d}\right\|_{L_{p}[a, b]^{d}} \\
& +C(p) \| \sum_{k=1}^{m}\left((-1)^{m-k}\left(\begin{array}{c}
m \\
k
\end{array}\right)\right)^{d} \frac{1}{k^{d}\left(\delta_{1} \ldots \delta_{d}\right)}\left(G\left(\left(x_{1}+k \delta_{1}, \ldots, x_{d}+k \delta_{d}\right)\right)\right. \\
& \left.-G\left(\left(x_{1}, \ldots, x_{d}\right)\right)\right) \|_{L_{p}[a, b]^{d}} \\
& \leq C(p) \omega_{m}\left(g,|\delta|,[a, b]^{d}\right)_{p}+C(p) \frac{2}{\left|\delta_{1}\right| \ldots\left|\delta_{d}\right|}\|G\|_{L_{p}[a, b]^{d}} \sum_{k=1}^{m}\left(\left(\begin{array}{c}
m \\
k
\end{array}\right) \frac{1}{k}\right)^{d} .
\end{aligned}
$$

Therefore

$$
\begin{aligned}
& E_{m-1}(f)_{L_{p}[a, b]^{d}} \leq\|g\|_{L_{p}[a, b]^{d}} \\
& \leq C(p) \omega_{m}\left(g,|\delta|,[a, b]^{d}\right)_{p}+C(p) \frac{2}{\left|\delta_{1}\right| \ldots\left|\delta_{d}\right|}\left(h_{1} \ldots h_{d}\right) \sum_{k=1}^{m}\left(\left(\begin{array}{c}
m \\
k
\end{array}\right) \frac{1}{k}\right)^{d} \omega_{m}\left(f, h,[a, b]^{d}\right)_{p},
\end{aligned}
$$

note that $\delta_{j}$ can always be chosen so that $h_{j} \geq\left|\delta_{j}\right| \geq h_{j} / 2$, then $E_{m-1}(f)_{L_{p}[a, b]^{d}} \leq$ $C(p) \omega_{m}\left(f, h,[a, b]^{d}\right)_{p}$

$$
+C(p) \frac{2}{\left(h_{1} \ldots h_{d}\right)}\left(h_{1} \ldots h_{d}\right) \sum_{k=1}^{m}\left(\left(\begin{array}{l}
m \\
k
\end{array}\right) \frac{1}{k}\right)^{d} \omega_{m}\left(f, h,[a, b]^{d}\right)_{p}
$$




$$
\begin{aligned}
& =\left(C(p)+2 C(p) \sum_{k=1}^{m}\left(\left(\begin{array}{c}
m \\
k
\end{array}\right) \frac{1}{k}\right)^{d}\right) \omega_{m}\left(f, h,[a, b]^{d}\right)_{p} \\
& =C(p, m, d) \omega_{m}\left(f, h,[a, b]^{d}\right)_{p},
\end{aligned}
$$

where $C(p, m, d)$ is Whitney constant

\section{References}

[1] H. Burkill , "Cesaro-Perron Almost Periodic Functions ", Proc. London Math. Soc., 3,150-174,1952.

[2] E. A. Storozhenko, "On The Approximation of Functions of Class $L_{p}, 0<p<1$, by Algebraic Polynomials ", Izv. Akad. Nauk SSSR Ser. Mat., 41,652-662. English Translation in Math. USSR Izv., 41, 1977.

[3] H. Whitney, "On Functions with $n-t h$ Bounded Differences ”, J. Math. Pures Appl., 36, 63-85,1957.

[4] H. Whitney, "On Bounded Functions with Bounded $n-t h$ Differences ", Proc. Amer. Math. Soc., $10,480-481,1959$.

[5] E. S. Bhaya , "On Constrained and Unconstrained Approximation", Ph. D dissertation, Baghdad University, College of Education Ibn Al - Haitham , 2003.

[6] S.Dekel and D. Leviatan, "Whitney Estimates for Convex Domains with Applications to Multivariate Piecewise Polynomial Approximation ”, Found. Comput. Math. 345- 368, 2004.

[7] Dinh Dung and Tino Ullrich, "On Whitney Type Inequalities for Local Anisotropic Polynomial Approximation ", 2011.

بر هنا في هذا البحث نظرية وتني لأفضل تقريب متعدد للدالة f التي تنتمي إلى الفضاء متعددة الحدود الجبرية متعددة المتغيرات الكلمات المفتاحية: نظرية وتتي, التقريب المتعدد, متعددة حدود لاكر انج • 